\title{
Therapeutic potential of the TWEAK/Fn14 pathway in intractable gastrointestinal cancer
}

\author{
RYO YORIKI $^{1 *}$, SATORU AKASHI $^{1 *}$, MASAYUKI SHO $^{1}$, TAKEO NOMI $^{1}$, ICHIRO YAMATO $^{1}$, \\ KIYOHIKO HOTTA ${ }^{1}$, TOMOYOSHI TAKAYAMA ${ }^{1}$, SOHEI MATSUMOTO ${ }^{1}$, KOHEI WAKATSUKI ${ }^{1}$, \\ KAZUHIRO MIGITA $^{1}$, HIDEO YAGITA ${ }^{2}$ and YOSHIYUKI NAKAJIMA ${ }^{1}$ \\ ${ }^{1}$ Department of Surgery, Nara Medical University, Nara; ${ }^{2}$ Department of Immunology, \\ Juntendo University School of Medicine, Tokyo, Japan
}

Received October 7, 2010; Accepted December 1, 2010

DOI: $10.3892 /$ etm.2010.181

\begin{abstract}
Tumor necrosis factor-like weak inducer of apoptosis (TWEAK) is a member of the TNF superfamily. It has been suggested that it plays a pivotal role in various physiological and pathological conditions due to its proinflammatory properties. Fibroblast growth-inducible 14 (Fn14) has been identified as a TWEAK receptor. A number of studies have suggested that TWEAK-Fn14 interaction results in the promotion of apoptosis, cell growth as well as angiogenesis. Although recent studies have indicated that TWEAK and Fn14 are expressed in a number of tumor lines and tissues, the therapeutic potential of this pathway has yet to be elucidated. This study investigated the potential of TWEAK and Fn14 in esophageal and pancreatic cancer as novel molecular targets for anti-cancer therapy. TWEAK and Fn14 protein expression was evaluated in 43 patients with esophageal cancer and 51 patients with pancreatic cancer by immunohistochemistry. As a result, either TWEAK or Fn14 expression was observed in $58.1 \%$ of the cases with esophageal cancer and $74.5 \%$ of the cases with pancreatic cancer. Furthermore, TWEAK/Fn14 gene expression was identified in the majority of the human esophageal and pancreatic cancer cell lines. Therapeutic efficacies of blocking TWEAK and Fn14 were evaluated by tumor growth inhibition assay in TWEAK- and Fn14-expressing human esophageal and pancreatic cancer cell lines. Coculture with anti-TWEAK or -Fn14 mAb was found to induce a $22-65 \%$ cell growth inhibition of these cells. Finally, the significant therapeutic effect of targeting this pathway under in vivo physiological conditions was confirmed using a murine
\end{abstract}

Correspondence to: Dr Masayuki Sho, Department of Surgery, Nara Medical University, 840 Shijo-cho, Kashihara, Nara 634-8522, Japan

E-mail: m-sho@naramed-u.ac.jp

*Contributed equally

Key words: esophageal cancer, pancreatic cancer, tumor necrosis factor-like weak inducer of apoptosis, fibroblast growth-inducible 14 , molecular target therapy gastrointestinal cancer model. In conclusion, the TWEAK/ Fn14 pathway may be functional and critical in intractable gastrointestinal cancers. Therefore, TWEAK and/or Fn14 may be novel molecular targets for anti-cancer therapy.

\section{Introduction}

Recent advances in cancer therapy have significantly improved patient prognosis (1). However, a number of gastrointestinal malignancies, including pancreatic and esophageal cancer, remain difficult to treat and are often fatal $(2,3)$. The development of a novel therapeutic approach against these intractable cancers is therefore urgently required. Molecular-targeted therapy may provide a breakthrough in cancer treatment. Although such novel approaches have improved the clinical outcome in several types of cancer, a definitive target for pancreatic and esophageal cancer has yet to be identified.

Tumor necrosis factor-like weak inducer of apoptosis (TWEAK, also known as CD255, TNFSF12 and APO3L) is a member of the TNF superfamily that was originally identified in 1997. It has been suggested that it plays a pivotal role in various inflammatory conditions due to its proinflammatory properties (4,5). Fibroblast growth factor-inducible 14 (Fn14, also known as CD266, TWEAKR and TNFRSF12A) was identified in 2001 as a TWEAK receptor that is linked to several intracellular signaling pathways (5-7). Previous studies have shown that TWEAK-Fn14 interaction promotes cell proliferation, migration, differentiation, apoptosis and angiogenesis as well as inflammation in various cell types $(8,9)$. Furthermore, it has been revealed that this pathway play a key role in a variety of diseases, including rheumatoid arthritis, multiple sclerosis and systemic lupus erythematosus (10-13). In addition, TWEAK and Fn14 may play roles in certain malignant tumors. Previous studies have demonstrated TWEAK expression in human malignancies in various organs, including the brain, lung, large intestine, liver and breast $(4,14,15)$. Fn14 expression has also been found in tumors in a variety of organs, including the liver, breast, brain, lung, pancreas and large intestine (16-19). Furthermore, a number of functional studies have suggested that TWEAK and/or Fn14 mediate tumor cell apoptosis, proliferation, migration, angiogenesis and survival $(20,21)$. Therefore, it has 
been proposed that TWEAK and Fn14 are potential targets for cancer therapy $(5,19,22)$. However, this strategy requires careful evaluation using in vitro and in vivo studies since TWEAK/Fn14 appears to exert diverse biological activities in humans $(5,23,24)$. In this study, we evaluated both TWEAK and Fn14 protein expression in actual human esophageal and pancreatic cancer tissues. We further examined the therapeutic potential of targeting the TWEAK/Fn14 pathway against gastrointestinal cancer in vitro and in vivo.

\section{Materials and methods}

Patients. A total of 43 patients with esophageal cancer and 51 with pancreatic cancer who underwent surgery at the Department of Surgery, Nara Medical University, were examined between 1995 and 2004. The esophageal cancers evaluated in this study were pathologically diagnosed as squamous cell carcinoma (SCC). The pancreatic cancers were diagnosed as invasive ductal carcinoma. For immunohistochemistry, each specimen was fixed in $10 \%$ phosphate-buffered formalin and embedded in paraffin. A serial section from each specimen was stained with H\&E for histological evaluation. The tumors were classified according to the TNM staging system. Written informed consent was obtained from the patients prior to the study according to our institutional guidelines.

Cell lines. Four human esophageal cancer cell lines, TE3 (highly differentiated squamous carcinoma), TE7 (adenocarcinoma), TE8 (moderately differentiated squamous carcinoma) and TE13 (poorly differentiated squamous carcinoma), and four human pancreatic cancer cell lines (CAPAN2, PK-8, PANC-1 and MIAPaCa-2) were obtained from the Cell Resource Center for Biomedical Research Institute of Development, Aging and Cancer, Tohoku University. A murine colorectal cancer cell line (colon-26) was obtained from the Riken Cell Bank (Ibaragi, Japan). The cell lines were maintained in Dulbecco's modified Eagle's medium/F12 medium with $10 \%$ heat-inactivated fetal bovine serum (FBS) at $37^{\circ} \mathrm{C}$ in a humidified atmosphere of $5 \% \mathrm{CO}_{2}$.

Reagents. For the in vitro and in vivo studies, anti-TWEAK blocking monoclonal antibodies (mAb) (CARL-1; as antihuman and MTW-1; as anti-murine) and anti-Fn14 blocking mAb (ITEM-2; as both anti-human and anti-murine) were prepared as previously described $(9,25,26)$.

Immunohistochemistry. The sections were stained using a Dako EnVision system (Dako Cytomation, Kyoto, Japan), according to the manual provided by the manufacturer. As primary antibodies, rabbit polyclonal anti-TWEAK (FL-249, 1:100 dilution; Santa Cruz Biotechnology Inc., Santa Cruz, CA, USA) and mouse monoclonal anti-Fn14 (Clone ITEM-4, 1:100 dilution; eBioscience, San Diego, CA, USA) were employed. After neutralization of endogenous peroxidase, sections were incubated for $90 \mathrm{~min}$ with primary antibodies. After three washes in PBS, the sections were incubated for $1 \mathrm{~h}$ with polymeric conjugate washed three times with phosphatebuffered saline (PBS). The reaction products were visualized with 3,3'-diaminobenzidine tetrahydrochloride and the slides were counterstained with hematoxylin.
RT-PCR analysis. Total RNA was isolated from the cell lines using guanidine isothiocyanate methods. RNA was transcribed to cDNA using Omniscript Reverse Transcriptase kit (Qiagen, Hilden, Germany) and oligo dT primers (Amersham Biosciences, Piscataway, NJ, USA). The RNA was heated for $5 \mathrm{~min}$ at $65^{\circ} \mathrm{C}$ and $4^{\circ} \mathrm{C}$ cooled on ice. Reaction buffer 10X $(2 \mu \mathrm{l}), 1 \mu \mathrm{l}$ of RNase inhibitor (10 U/ $\mu \mathrm{l}), 2 \mu \mathrm{l}$ of a mixture of dNTPs, $1 \mu \mathrm{l}$ of oligodT primers $(10 \mu \mathrm{M}), 1 \mu \mathrm{l}$ of Omniscript Reverse Transcriptase (4 U/ $\mu \mathrm{l})$ and RNase-free water were added for a total volume of $20 \mu 1$. The mixture was incubated for $60 \mathrm{~min}$ at $37^{\circ} \mathrm{C}$. After incubation, the cDNA was stored at $-80^{\circ} \mathrm{C}$ before analysis. The cDNA solution $(0.5 \mu \mathrm{l})$ was amplified in a total volume of $20 \mu \mathrm{l}$ that contained $2 \mu \mathrm{l}$ of 10X PCR buffer, $0.6 \mu \mathrm{l}$ of $\mathrm{MgCl}_{2}(50 \mathrm{mM}), 0.2 \mu \mathrm{l}$ of Platinum Taq DNA Polymerase (Invitrogen, Carlsbad, CA, USA), sense primer $(50 \mu \mathrm{M})$, antisense primer $(50 \mu \mathrm{M})$ and $2 \mu 1$ of a mixture of dNTPs (Takara, Ohtsu, Japan). The amplification involved an initial step of denaturation at $94^{\circ} \mathrm{C}$ for $3 \mathrm{~min}$, followed by cycles consisting of denaturation at $94^{\circ} \mathrm{C}$ for $1 \mathrm{~min}$, annealing at $63^{\circ} \mathrm{C}$ for $1 \mathrm{~min}$, and chain elongation at $72^{\circ} \mathrm{C}$ for $1 \mathrm{~min}$. The sequences of sense and antisense primers, and the product size for TWEAK, Fn14 and $\beta$-actin were: TWEAK, sense 5'-CCCTGCGCTGCCTGGAGGAA-3' and antisense 5'-AGA CCAGGGCCCCTCAGTGA-3' (amplicon size 200 bp); Fn14, sense 5'-CCAAGCTCCTCCAACCACAA-3' and antisense 5'-TGGGGCCTAGTGTCAAGTCT-3' (amplicon size 242 bp); $\beta$-actin, sense 5'-ATCAAGATCCTGACCGAGCG-3' and antisense 5'-TACTTGCGCTCAGGAGGAGC-3'. The amplified products were analyzed by electrophoresis of the PCR product on a $2 \%$ agarose gel plus ethidium bromide in TBE buffer.

Cell growth inhibition assay. Human cancer cells were added at a concentration of $1 \times 10^{3}$ cells in $100 \mu \mathrm{l}$ to the individual wells of 96-well flat-bottomed microtiter plates. Then, $25 \mu \mathrm{g} /$ ml of anti-human TWEAK mAb (CARL-1), anti-Fn14 mAb (ITEM-2) and control IgG were added to each well every other day and were further incubated at $37^{\circ} \mathrm{C}$ in a humidified atmosphere of $5 \% \mathrm{CO}_{2}$. After 3 or 7 days, the cells were rinsed with PBS, fixed with 5\% glutaraldehyde (Nacalai Tesque, Kyoto, Japan) in PBS and then stained with $0.2 \%$ crystal violet (Wako, Osaka, Japan) and 100 mM CAPS (Wako). The absorbance of each well was measured at $540 \mathrm{~nm}$ with Multiskan MS (Labsystems, Helsinki, Finland). The growth inhibition rate was calculated using the following formula: $\%$ of growth inhibition rate $=$ absorbance of mAb-treated cells - spontaneous absorbance/absorbance of control cells - spontaneous absorbance. The samples were examined in triplicate.

Animal study. Female BALB/c mice (8 weeks old) were obtained from Clea Japan (Tokyo, Japan). The mice were maintained under specific pathogen-free conditions in the animal facility at Nara Medical University. The experiments were conducted under a protocol approved by our institutional review board. Cells ( $1 \times 10^{6}$ murine colorectal adenocarcinoma colon-26) were subcutaneously inoculated on one side of the ventral surface in the lower flank region of syngeneic BALB/c mice. Treatment was started 7 days later when a small palpable lump was evident, ranging from 4 to $6 \mathrm{~mm}$ in diameter. Some mice were intraperitoneally injected with $0.3 \mathrm{mg}$ of each $\mathrm{mAb}$ every other day for 3 weeks. The control mice received control 

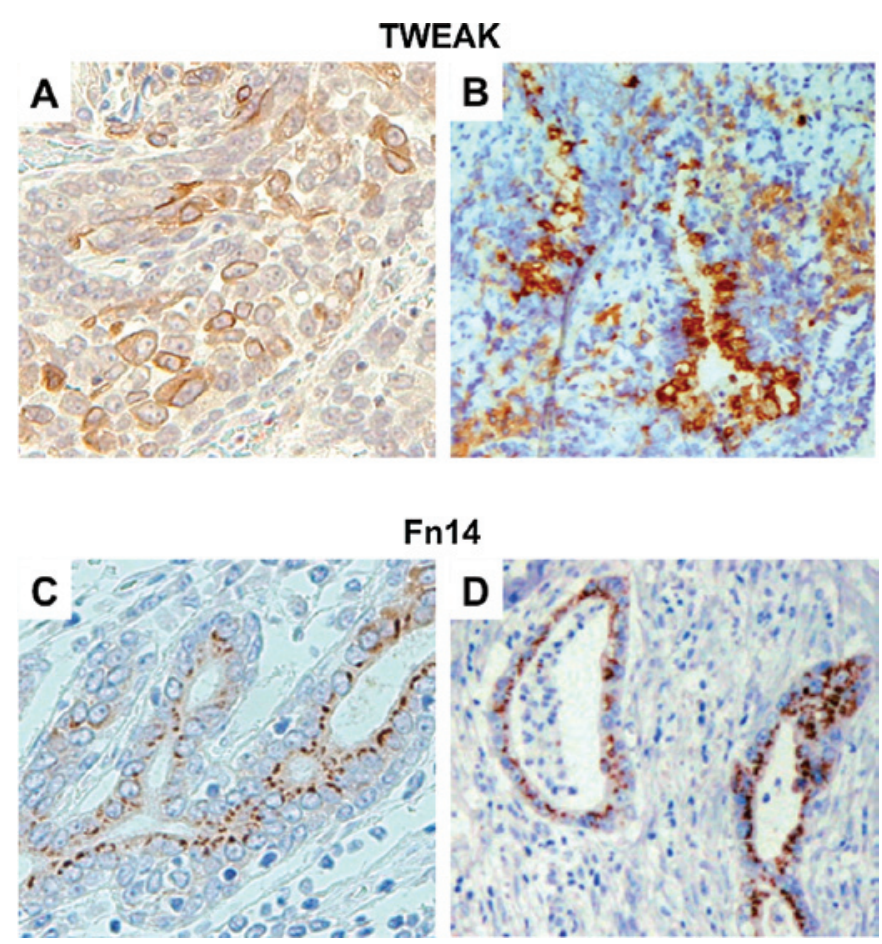

Figure 1. Immunohistochemical staining of human esophageal and pancreatic cancer tissues with FL-249 recognizing (top) TWEAK and ITEM-4 recognizing (bottom) Fn14. (A) Representative case of TWEAK-positive esophageal cancer, (B) TWEAK-positive pancreatic cancer, (C) Fn14positive esophageal cancer and (D) Fn14-positive pancreatic cancer. Original magnification, $\mathrm{x} 200$

IgG. The tumor size was determined by caliper measurements. The tumor volume was calculated according to the formula: $\mathrm{V}=\mathrm{A} \times \mathrm{B}^{2} / 2\left(\mathrm{~mm}^{3}\right)$, where $\mathrm{A}$ is the largest diameter $(\mathrm{mm})$ and $\mathrm{B}$ is the smallest diameter $(\mathrm{mm})$.

Statistical analysis. The experimental data of the growth inhibition assay were analyzed by the Student's t-test. Differences of $\mathrm{P}<0.05$ were considered to indicate statistical significance.

\section{Results}

TWEAK and Fn14 expression in human esophageal and pancreatic cancer. TWEAK and Fn14 protein expression in actual human esophageal cancer and pancreatic cancer tissues was examined by immunohistochemical staining. In both cancer tissues, TWEAK expression was identified in the cytoplasm and membrane of cancer cells, and Fn14 was observed mainly in the tumor cellular membrane (Fig. 1). In esophageal cancer, $20 / 43$ cases $(46.5 \%)$ stained positively for TWEAK and 10 cases (23.2\%) were positive for Fn14. Overall, either TWEAK or Fn14 expression was observed in 25 cases $(58.1 \%)$ (Table I). In pancreatic cancer, 18/51 (35.3\%) stained positively for TWEAK and 35 (68.6\%) were positive for Fn14. Furthermore, 15 (29.4\%) cases showed positive staining for both TWEAK and Fn14. Overall, either TWEAK or Fn14 expression was observed in 38 cases (74.5\%) (Table II).

TWEAK and Fn14 mRNA expression in human esophageal and pancreatic cancer cell lines. The gene expression of TWEAK and Fn14 was examined in four cell lines of each cancer type.
Table I. Correlation between TWEAK and Fn14 expression in esophageal cancer.

\begin{tabular}{lrcrc}
\hline & \multicolumn{3}{c}{ TWEAK } \\
\cline { 2 - 5 } & Positive (\%) & Negative (\%) & Total (\%) \\
\hline Fn14 & $5(11.6)$ & $5(11.6)$ & $10(23.2)$ \\
Positive & $15(34.9)$ & $18(41.9)$ & $33(76.8)$ \\
Negative & $20(46.5)$ & $23(53.5)$ & $43(100.0)$ \\
Total & & &
\end{tabular}

Table II. Correlation between TWEAK and Fn14 expression in pancreatic cancer.

\begin{tabular}{lrlll}
\hline & \multicolumn{4}{c}{ TWEAK } \\
\cline { 2 - 5 } & Positive (\%) & Negative (\%) & Total (\%) \\
\hline Fn14 & $15(29.4)$ & $20(39.2)$ & $35(68.6)$ \\
Positive & $3(5.9)$ & $13(25.5)$ & $16(31.4)$ \\
Negative & $18(35.3)$ & $33(64.7)$ & $51(100.0)$ \\
Total & & & \\
\hline
\end{tabular}

As a result, RT-PCR analysis indicated that both TWEAK and Fn14 were detected in all tested esophageal cell lines. In human pancreatic cancer cell lines, TWEAK was expressed in 3/4 cell lines (CAPAN2, PANC-1 and MIAPaCa-2), and Fn14 was observed in all the cell lines (Fig. 2).

Blockade of the TWEAK/Fn14 pathway inhibits gastrointestinal tumor growth. For the clinical application of targeting TWEA/Fn14 pathway, the therapeutic efficacy of its blockade on gastrointestinal cancer cell growth was evaluated using a number of TWEAK- or Fn14-expressing human cancer cell lines. After co-culture with mAb, TWEAK blockade had an inhibitory effect on both esophageal and pancreatic cancer growth (Fig. 3). The reduction rates of cell growth on the 7th day were: TE3, 22\%; TE7, 38\%; CAPAN2, 53\%; PANC-1, 47\%; and MIAPaCa-2, 47\%. Furthermore, the Fn14 blockade exerted a similar effect on the cell growth of both types of cancer (reduction rate: TE3, 52\%; TE7, 50\%; CAPAN2, 55\%; PANC-1, 49\% and MIAPaCa-2: 65\%) (Fig. 4).

TWEAK/Fn14 blockade inhibits gastrointestinal cancer growth in vivo. In order to confirm the therapeutic efficacy of targeting TWEAK/Fn14 in gastrointestinal cancer, a physiological in vivo model was employed. To this end, using the murine colorectal adenocarcinoma colon-26 cell line, the efficacy of TWEAK/Fn14 blockade in wild-type BALB/c mice was evaluated. The treatment of anti-TWEAK or -Fn14 blocking mAb significantly inhibited tumor growth in the treated mice (Fig. 5). Thus, the beneficial effect of either TWEAK or Fn14 blockade on gastrointestinal cancer was confirmed even in immunocompetent mice. 


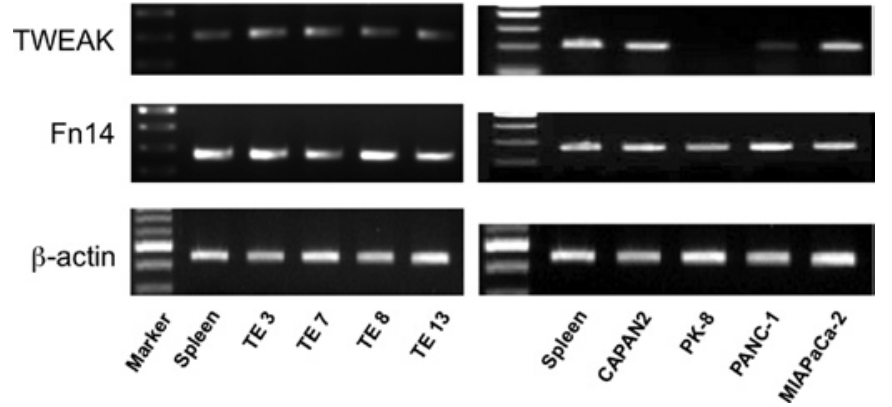

Figure 2. TWEAK and Fn14 mRNA expression in human esophageal and pancreatic cancer cell lines examined by reverse transcriptase-PCR. A 200-bp human TWEAK sequence, a 242-bp human Fn14 sequence and a $\beta$-actin sequence were amplified from total RNA, separated by agarose gel electrophoresis and visualized by ethidium bromide staining.

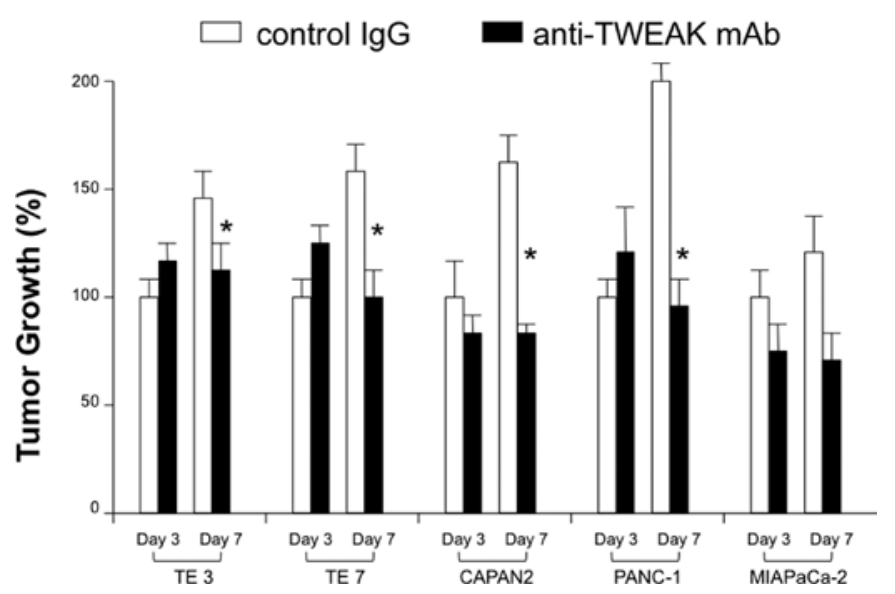

Figure 3. Growth inhibition of esophageal and pancreatic cell lines by antiTWEAK mAb $(25 \mathrm{mg} / \mathrm{ml})$. The cancer cells were co-cultured with either control IgG (white boxes) or anti-TWEAK mAb (black boxes). "Indicates statistical difference in the growth of tumor cells treated with anti-TWEAK $\mathrm{mAb}$ compared to the controls. The results are expressed as the mean number of three wells \pm SD obtained from one representative of three individual experiments.

\section{Discussion}

TWEAK is a multifunctional cytokine that controls various cellular activities. TWEAK functions by binding the cell-surface receptor, Fn14. Recent cumulative evidence has revealed that the TWEAK/Fn14 pathway regulates a variety of physiological and pathological conditions (5). Although it has been suggested that this pathway is involved in tumor development and progression, the therapeutic potential of targeting TWEAK/Fn14 has yet to be elucidated (14-16,19,22,27). In this study, we evaluated the clinical usefulness of TWEAK/ Fn14-targeted therapy in esophageal and pancreatic cancer in representative intractable gastrointestinal tumors.

First, we examined the protein expression of TWEAK/ Fn14 in actual human cancer tissues. We confirmed this expression in approximately $60 \%$ of esophageal and $75 \%$ of pancreatic cancer cases. Although TWEAK protein expression has not been previously evaluated in esophageal and pancreatic cancer, it was recently reported that Fn14 expression is positive in $27 \%$ of esophageal cancer and $60 \%$ of pancreatic

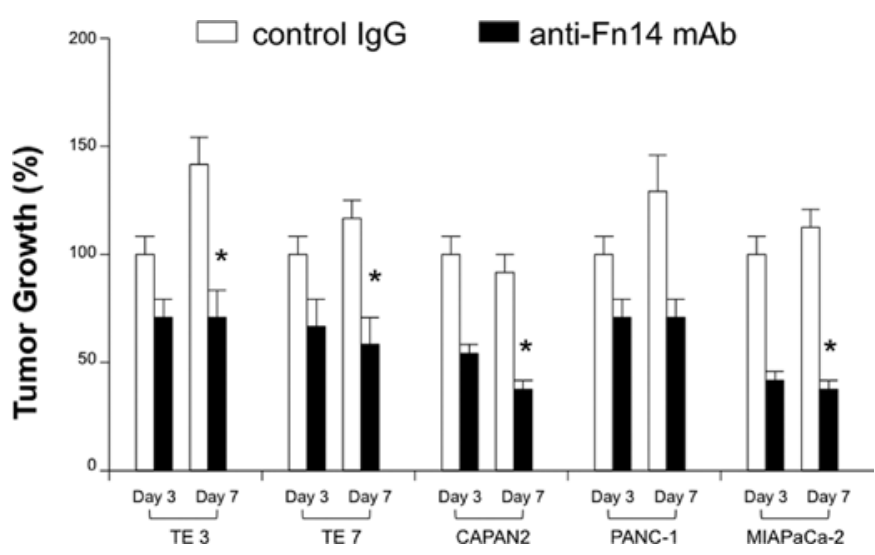

Figure 4. Growth inhibition of esophageal and pancreatic cell lines by antiFn $14 \mathrm{mAb}(25 \mathrm{mg} / \mathrm{ml})$. The cancer cells were co-cultured with either control IgG (open boxes) or anti-Fn14 mAb (closed boxes). "Indicates statistical difference in the growth of tumor cells treated with anti-Fn14 mAb compared to the controls. The results are expressed as the mean number of three wells \pm SD obtained from one representative of three individual experiments.

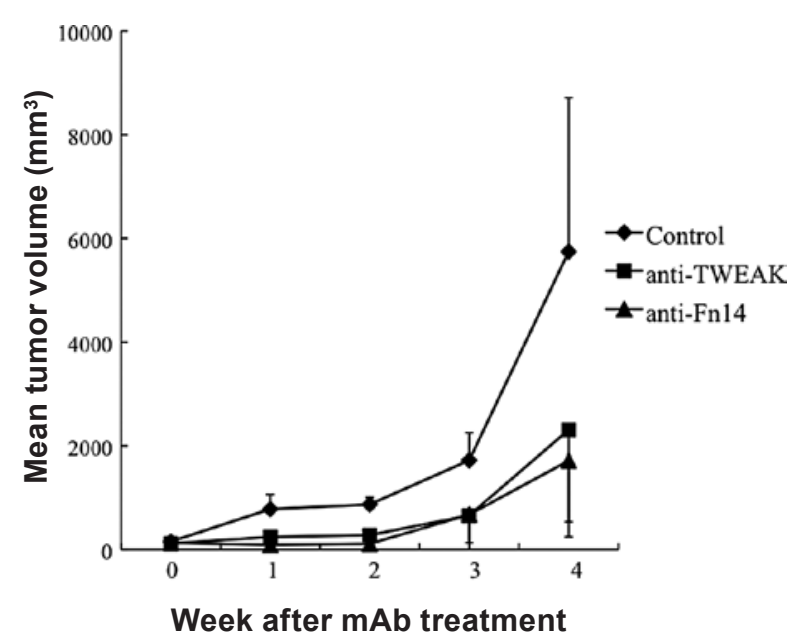

Figure 5. Therapeutic efficacy of targeting TWEAK/Fn14 in gastrointestinal cancer. The effect of TWEAK/Fn14 blockade on gastrointestinal cancer was examined in vivo. Colon-26 was implanted in the flank of the syngeneic $\mathrm{BALB} / \mathrm{c}$ mice. The mice were treated with $0.3 \mathrm{mg}$ of each $\mathrm{mAb}$ every other day for 3 weeks. The control mice received control IgG. The mean tumor volume at 1,2, 3 and 4 weeks after starting treatment was; anti-TWEAK $\mathrm{mAb}, 243,279,656$ and $2,311 \mathrm{~mm}^{3}$, respectively; anti-Fn14 mAb, 91, 111, 676 and $1,713 \mathrm{~mm}^{3}$, respectively; control, 781, 870, 1,722 and 5,747 $\mathrm{mm}^{3}$, respectively. $n=6$ for each group. Data points, mean tumor size; bars, SD. Either TWEAK or Fn14 blockade inhibited tumor growth compared to the controls. The statistical differences were; TWEAK blockade, $0.005,<0.0001$, 0.02 and 0.07 at $1,2,3$ and 4 weeks, respectively, compared to the control; Fn14 blockade, $0.0007,<0.0001,0.06$ and 0.02 , respectively, compared to the control.

cancer cases (19). In our study, it was positive in 23 and $68 \%$, respectively. These data appear similar, even though there are racial and tumor histological differences. Furthermore, most of the esophageal and pancreatic cancer cell lines examined in this study demonstrated both TWEAK and Fn14 expression. Notably, Fn14 mRNA expression was recently reported as one of the best markers for the early diagnosis of esophageal cancer (28). The TWEAk/Fn14 pathway is therefore a promising novel target for intractable gastrointestinal cancer. 
Next, we investigated the therapeutic potential of targeting the TWEAK/Fn14 pathway in gastrointestinal cancer both in vitro and in vivo. We found that TWEAK and Fn14 blockade had a substantial effect on the inhibition of gastrointestinal cancer. Our in vitro experiments clearly demonstrated that TWEAK and Fn14 blockade significantly inhibited cancer cell growth. Thus, data indicate that both molecules are directly involved in the tumor proliferation of esophageal and pancreatic cancer. Furthermore, in vivo data evaluated in physiological conditions also demonstrate that TWEAK and/ or Fn14 are functional and may be potential targets for a novel anti-cancer therapy. However, due to the diverse functions of TWEAK/Fn14, there may be a number of caveats for clinical application as a molecular-targeted therapy. Tumor cell apoptosis is essential for the induction of therapeutic efficacy in a variety of cancer treatments. TWEAK/Fn14 has been found to be a critical regulator to promote the apoptosis of certain cells under various circumstances $(4,5,29)$. Therefore, its blockade may result in the promotion of resistance to treatment-related apoptosis of tumor cells. Furthermore, recent studies have suggested that TWEAK/Fn14 plays a critical role in tissue repair and regeneration (18,30-32). Therefore, inhibition of this pathway may have detrimental effects or be harmful for cancer patients.

On the other hand, accumulating evidence suggests the therapeutic potential of TWEAK and/or Fn14 blockade as a novel anti-cancer therapy. Soluble TWEAK has previously been shown to promote tumor growth and angiogenesis (21). In addition, Fn14 overexpression has been demonstrated to induce tumor cell migration (20,22). Furthermore, it has been shown that tumor invasion was significantly inhibited by Fn14 blockade using the RNA interference method (16,31). More recently, newly developed antibodies for targeting Fn14 have been demonstrated to inhibit tumor growth through direct inhibition and antibody-dependent cellular cytotoxicity (19). Our in vitro and in vivo therapeutic data further support a potential of blocking the TWEAK/Fn14 pathway in cancer treatment. A number of potential underlying mechanisms are involved in anti-TWEAK and anti-Fn14 mAb treatment. These include direct inhibition of proliferation, migration, apoptosis, angiogenesis and survival of tumor cells $(5,22)$. Also, antibody-dependent mechanisms may play a critical part in vivo, in clinically relevant physiological conditions (19). Further studies are required to clarify the possible mechanisms of the antitumor effect induced by targeting TWEAK/ Fn14 in intractable gastrointestinal cancer.

It is crucial to evaluate the combination therapy of targeting TWEAK and Fn14 with conventional antitumor treatments, including chemotherapy and radiotherapy, since chemotherapy and radiotherapy influence the multifunction of TWEAK and Fn14, leading to either a synergistic or detrimental antitumor effect. Particularly in intractable gastrointestinal tumors including esophageal and pancreatic cancer, multimodal treatment appears to be essential for improving patient prognosis. Therefore, further research is warranted to evaluate the combination therapy with current standard chemotherapy, since new molecular-targeted therapies have been reported to enhance the clinical outcome of conventional chemotherapy even in intractable cancer (33). To date, no studies address these issues in regard to the TWEAK/Fn14 pathway.
In conclusion, we demonstrated TWEAK/Fn14 expression in human esophageal and pancreatic cancers representative of intractable gastrointestinal malignancies. Our data indicate that the TWEAK/Fn14 pathway is a novel molecular target for anti-cancer therapy and may provide the rationale for developing this novel strategy against such diseases.

\section{Acknowledgements}

This study was supported by the following grants: Grantsin-Aid for Scientific Research from the Ministry of Education, Culture, Sports, Science and Technology of Japan, nos. 19591491 and 21591648; Research Grant from the Pancreas Research Foundation of Japan; Research Grant from the Foundation for Promotion of Cancer Research in Japan; Research Grant from the Daiwa Securities Health Foundation; and Research Grant from the Nakayama Cancer Research Institute (to M. Sho).

\section{References}

1. Jemal A, Siegel R, Xu J and Ward E: Cancer statistics, 2010. CA Cancer J Clin 60: 277-300, 2010.

2. Kamangar F, Dores GM and Anderson WF: Patterns of cancer incidence, mortality, and prevalence across five continents: defining priorities to reduce cancer disparities in different geographic regions of the world. J Clin Oncol 24: 2137-2150, 2006 .

3. Li D, Xie K, Wolff R and Abbruzzese JL: Pancreatic cancer. Lancet 363: 1049-1057, 2004

4. Chicheportiche Y, Bourdon PR, Xu H, Hsu YM, Scott H, Hession C, Garcia I and Browning JL: TWEAK, a new secreted ligand in the tumor necrosis factor family that weakly induces apoptosis. J Biol Chem 272: 32401-32410, 1997.

5. Winkles JA: The TWEAK-Fn14 cytokine-receptor axis: discovery, biology and therapeutic targeting. Nat Rev Drug Discov 7: 411-425, 2008.

6. Wiley SR, Cassiano L, Lofton T, Davis-Smith T, Winkles JA, Lindner V, Liu H, Daniel TO, Smith CA and Fanslow WC: A novel TNF receptor family member binds TWEAK and is implicated in angiogenesis. Immunity 15: 837-846, 2001.

7. Kumar M, Makonchuk DY,Li H, Mittal A and Kumar A: TNF-like weak inducer of apoptosis (TWEAK) activates proinflammatory signaling pathways and gene expression through the activation of TGF-beta-activated kinase 1. J Immunol 182: 2439-2448, 2009.

8. Polek TC, Talpaz M, Darnay BG and Spivak-Kroizman T: TWEAK mediates signal transduction and differentiation of RAW264.7 cells in the absence of Fn14/TweakR. Evidence for a second TWEAK receptor. J Biol Chem 278: 32317-32323, 2003.

9. Harada N, Nakayama M, Nakano H, Fukuchi Y, Yagita H and Okumura K: Pro-inflammatory effect of TWEAK/Fn14 interaction on human umbilical vein endothelial cells. Biochem Biophys Res Commun 299: 488-493, 2002.

10. Zhao Z, Burkly LC, Campbell S, Schwartz N, Molano A, Choudhury A, Eisenberg RA, Michaelson JS and Putterman C: TWEAK/Fn14 interactions are instrumental in the pathogenesis of nephritis in the chronic graft-versus-host model of systemic lupus erythematosus. J Immunol 179: 7949-7958, 2007.

11. Desplat-Jego S, Creidy R, Varriale S, Allaire N, Luo Y, Bernard D, Hahm K, Burkly L and Boucraut J: Anti-TWEAK monoclonal antibodies reduce immune cell infiltration in the central nervous system and severity of experimental autoimmune encephalomyelitis. Clin Immunol 117: 15-23, 2005.

12. Perper SJ, Browning B, Burkly LC, et al: TWEAK is a novel arthritogenic mediator. J Immunol 177: 2610-2620, 2006.

13. Kamata K, Kamijo S, Nakajima A, Koyanagi A, Kurosawa H, Yagita $\mathrm{H}$ and Okumura K: Involvement of TNF-like weak inducer of apoptosis in the pathogenesis of collagen-induced arthritis. J Immunol 177: 6433-6439, 2006.

14. Kawakita T, Shiraki K, Yamanaka Y, Yamaguchi Y, Saitou Y, Enokimura N, Yamamoto N, Okano H, Sugimoto K, Murata K and Nakano T: Functional expression of TWEAK in human colonic adenocarcinoma cells. Int J Oncol 26: 87-93, 2005. 
15. Kawakita T, Shiraki K, Yamanaka Y, Yamaguchi Y, Saitou Y, Enokimura N, Yamamoto N, Okano H, Sugimoto K, Murata K and Nakano T: Functional expression of TWEAK in human hepatocellular carcinoma: possible implication in cell proliferation and tumor angiogenesis. Biochem Biophys Res Commun 318: 726-733, 2004.

16. Tran NL, McDonough WS, Savitch BA, et al: Increased fibroblast growth factor-inducible 14 expression levels promote glioma cell invasion via Rac1 and nuclear factor-kappaB and correlate with poor patient outcome. Cancer Res 66: 9535-9542, 2006.

17. Han H, Bearss DJ, Browne LW, Calaluce R, Nagle RB and von Hoff DD: Identification of differentially expressed genes in pancreatic cancer cells using cDNA microarray. Cancer Res 62 2890-2896, 2002.

18. Feng SL, Guo Y, Factor VM, Thorgeirsson SS, Bell DW, Testa JR, Peifley KA and Winkles JA: The Fn14 immediate-early response gene is induced during liver regeneration and highly expressed in both human and murine hepatocellular carcinomas. Am J Pathol 156: 1253-1261, 2000.

19. Culp PA, Choi D, Zhang Y, et al: Antibodies to TWEAK receptor inhibit human tumor growth through dual mechanisms. Clin Cancer Res 16: 497-508, 2010.

20. Tran NL, McDonough WS, Savitch BA, Sawyer TF, Winkles JA and Berens ME: The tumor necrosis factor-like weak inducer of apoptosis (TWEAK)-fibroblast growth factor-inducible 14 (Fn14) signaling system regulates glioma cell survival via NFkappaB pathway activation and BCL-XL/BCL-W expression. J Biol Chem 280: 3483-3492, 2005.

21. Ho DH, Vu H, Brown SA, Donohue PJ, Hanscom HN and Winkles JA: Soluble tumor necrosis factor-like weak inducer of apoptosis overexpression in HEK293 cells promotes tumor growth and angiogenesis in athymic nude mice. Cancer Res 64: 8968-8972, 2004.

22. Winkles JA, Tran NL and Berens ME: TWEAK and Fn14: new molecular targets for cancer therapy? Cancer Lett 235: 11-17, 2006.

23. Polavarapu R, Gongora MC, Winkles JA and Yepes M: Tumor necrosis factor-like weak inducer of apoptosis increases the permeability of the neurovascular unit through nuclear factorkappa B pathway activation. J Neurosci 25: 10094-10100, 2005.
24. Potrovita I, Zhang W, Burkly L, Hahm K, Lincecum J, Wang MZ, Maurer MH, Rossner M, Schneider A and Schwaninger M: Tumor necrosis factor-like weak inducer of apoptosis-induced neurodegeneration. J Neurosci 24: 8237-8244, 2004.

25. Nakayama $M$, Harada $N$, Okumura $K$ and Yagita $H$ : Characterization of murine TWEAK and its receptor (Fn14) by monoclonal antibodies. Biochem Biophys Res Commun 306: $819-825,2003$

26. Nakayama M, Ishidoh K, Kojima Y, Harada N, Kominami E, Okumura $\mathrm{K}$ and Yagita $\mathrm{H}$ : Fibroblast growth factor-inducible 14 mediates multiple pathways of TWEAK-induced cell death. J Immunol 170: 341-348, 2003 .

27. Han S, Yoon K, Lee K, Kim K, Jang H, Lee NK, Hwang K and Young Lee S: TNF-related weak inducer of apoptosis receptor, a TNF receptor superfamily member, activates NF-kappa B through TNF receptor-associated factors. Biochem Biophys Res Commun 305: 789-796, 2003.

28. Watts GS, Tran NL, Berens ME, Bhattacharyya AK, Nelson MA, Montgomery EA and Sampliner RE: Identification of Fn14/ TWEAK receptor as a potential therapeutic target in esophageal adenocarcinoma. Int J Cancer 121: 2132-2139, 2007.

29. Wang D, Fung JN, Tuo Y, Hu L and Chen C: TWEAK/Fn14 promotes apoptosis of human endometrial cancer cells via caspase pathway. Cancer Lett 294: 91-100, 2010.

30. Jakubowski A, Ambrose C, Parr M, Lincecum JM, Wang MZ, Zheng TS, Browning B, Michaelson JS, Baetscher M, Wang B, Bissell DM and Burkly LC: TWEAK induces liver progenitor cell proliferation. J Clin Invest 115: 2330-2340, 2005.

31. Tanabe K, Bonilla I, Winkles JA and Strittmatter SM: Fibroblast growth factor-inducible-14 is induced in axotomized neurons and promotes neurite outgrowth. J Neurosci 23: 9675-9686, 2003.

32. Girgenrath M, Weng S, Kostek CA, et al: TWEAK, via its receptor Fn14, is a novel regulator of mesenchymal progenitor cells and skeletal muscle regeneration. EMBO J 25: 5826-5839, 2006.

33. Moore MJ, Goldstein D, Hamm J, et al: Erlotinib plus gemcitabine compared with gemcitabine alone in patients with advanced pancreatic cancer: a phase III trial of the National Cancer Institute of Canada Clinical Trials Group. J Clin Oncol 25: 1960-1966, 2007. 